\title{
Atomic and Molecular Structure Calculations for Superheavy Elements
}

\author{
B. Fricke*,a and V. Pershina ${ }^{\mathrm{b}}$ \\ ${ }^{a}$ Department of Physics, University of Kassel, D-34109 Kassel, Germany \\ ${ }^{\mathrm{b}}$ Gesellschaft für Schwerionenforschung, D-64220 Darmstadt, Germany
}

\section{Received: November 13, 2001; In Final Form: April 12, 2002}

\begin{abstract}
A first prediction of the chemistry of superheavy elements has been given 30 years ago using many-electron relativistic atomic structure calculations in the quality of that time. ${ }^{1}$ The first relativistic molecular calculation for a system with a transactinide element was published in $1977 .^{2}$ Since then relativistic atomic and molecular structure calculations have been considerably improved and applied to many atoms and molecules. In this review we try to outline the general theory and the development of the quality of such calculations with less and less approximations as well as better and better numerical methods and higher accuracy. But even if one has quite accurate total energies, quantities like effective charges, overlap populations or radii of the outermost wavefunctions are still a very valuable information for an effective prediction of the chemical behavior of the superheavy elements.
\end{abstract}

\section{Introduction}

The structure of the heavy elements has attracted much interest since the earlier days of quantum mechanics. It was soon realized that the chemical behavior is a result of the structure of the outer valence electrons of the atoms. The discovery and availability of the actinides and transactinides in physical and chemical research started this field in the mid forties. Theoretical methods became available in the early sixties which allowed to predict the electronic structure for very heavy elements. Nowadays, the analysis of many physical and chemical experiments as well as a series of computations has lead to a considerable understanding of these elements. Today the predictive power is so enhanced that we are relatively sure about the magnitude of most of the physical quantities and of the chemical behavior of these elements, which are either just observed on a single atom basis ( $Z=107$ to 112$)$ or will hopefully be observed in the region of the superheavy elements in the vicinity of the nuclear charge 114 in the future.

\section{General Theory}

We all believe in the Schrödinger- (or Dirac-) equation

$$
\hat{H}|\Psi\rangle=-i \frac{\partial}{\partial t}|\Psi\rangle
$$

respectively (in the time-independent form)

$$
\hat{H}|\Psi\rangle=E|\Psi\rangle \text {. }
$$

In order to solve this equation, one has to follow various steps:

- At first one has to define the Hamiltonian which one would like to solve.

- Secondly, one has to separate the nuclear and electronic coordinates with the help of the Born-Oppenheimer approximation and

- thirdly, choose an ansatz for the wavefunction $\Psi$.

2.1. Choice of the Hamiltonian. As a first step in all "normal" calculations only the Coulomb interaction between all charged particles usually is taken into account.

$$
\begin{aligned}
\hat{H} \cong & \sum_{\alpha=1}^{N}\{\frac{\nabla_{\alpha}^{2}}{2 M}+\sum_{i=1}^{Z} \frac{\nabla_{\alpha_{i}}^{2}}{2 m}+\underbrace{\sum_{\beta=1}^{N}\left[\frac{1}{2} \frac{(Z e)^{2}}{\left|R_{\alpha}-R_{\beta}\right|}\right.}_{V_{\alpha \beta}} \\
& +\underbrace{\sum_{i=1}^{Z}\left(-\frac{Z e^{2}}{\left|r_{\beta_{i}}-R_{\alpha}\right|}\right.}_{V_{\alpha i}}+\underbrace{\left.\left.\left.\frac{1}{2} \sum_{j=1}^{Z} \frac{e^{2}}{\left|r_{\alpha_{i}}-r_{\beta_{j}}\right|}\right)\right]\right\}}_{V_{i j}} .
\end{aligned}
$$

*Corresponding author. E-mail: fricke@ physik.uni-kassel.de. FAX: $+49561804-4006$
As the next step the interaction between the magnetic moments of the spins and orbital angular momenta of the electrons (and nuclei) are taken care of (usually in the form of the Breit interaction). Finally in the most advanced form the interaction due to quantum electrodynamical effects will be considered.

2.2. Separation of Nuclear and Electronic Coordinates. The Born-Oppenheimer ansatz for the total wavefunction

$$
\Psi(R, r)=\psi_{e}(R, r) \cdot \psi_{N, e}(R)
$$

allows to separate eq 1 in two equations. ( $R$ stands for the nuclear coordinates, $r$ for the electronic coordinates, the index $e$ is the electronic quantum number, and the index $N$ is the nuclear quantum number.)

$$
\begin{gathered}
\sum_{i} \frac{\nabla_{i}^{2}}{2 m} \psi_{e}(R, r)+\left[E_{e}(r)-V_{i j}-V_{\alpha i}\right] \psi_{e}(R, r)=0, \\
\sum_{\alpha} \frac{\nabla_{\alpha}}{M} \psi_{N, e}(R)+\left[E-E_{e}(r)-V_{\alpha \beta}\right] \psi_{N, e}(R)=0 .
\end{gathered}
$$

Equation 4 is the basis for Quantum Chemistry, and eq 5 is the equation for the nuclear motion which is the basis for Molecular Dynamics.

2.3. Choice of a Wavefunction. The simplest approach is a product of single particle functions $\psi=\Pi_{i} \varphi_{i}$ which is, however, not antisymmetric (Hartree approximation). Another possibility is the ansatz of a Slater determinant

$$
\psi=\frac{1}{\sqrt{n !}}\left|\begin{array}{cccc}
\phi_{1}(1) & \phi_{2}(1) & \ldots & \phi_{n}(1) \\
\phi_{1}(2) & \phi_{2}(2) & \ldots & \phi_{n}(2) \\
\vdots & \vdots & & \vdots \\
\phi_{1}(n) & \phi_{2}(n) & \ldots & \phi_{n}(n)
\end{array}\right|
$$

This is an antisymmetric ansatz, and the Pauli principle is automatically included. If the ansatz 6 is plugged into eq 4, one gets after a variational procedure the Hartree-Fock equations

$$
\begin{gathered}
-\frac{\nabla_{i}^{2}}{2 m}-\frac{Z}{r}+\left[\sum_{k=1}^{n} \int \phi_{k}^{*}\left(\vec{r}_{i}^{\prime}\right) \phi_{k}\left(\vec{r}_{j}^{\prime}\right) \frac{e^{2}}{\left(\vec{r}-\vec{r}^{\prime}\right)} d \vec{r}^{\prime}\right] \phi_{i}(\vec{r}) \\
\text { Coulomb Part, } \\
-\sum_{k=1}^{n}\left[\int \phi_{k}^{*}\left(\vec{r}^{\prime}\right) \phi_{i}\left(\vec{r}^{\prime}\right) \frac{e^{2}}{\left|\vec{r}-\vec{r}^{\prime}\right|} d \vec{r}^{\prime}\right] \phi_{k}(\vec{r})=\varepsilon_{i} \phi_{i}(\vec{r}) \\
\text { Exchange Part. }
\end{gathered}
$$

Equation 7 is solved iteratively in a self-consistent field (SCF) way. The wavefunctions in eq 7 have to be specified as atomic 
TABLE 1: Results from atomic structure calculations for the elements 104-108.

\begin{tabular}{|c|c|c|c|c|c|c|}
\hline \multirow[t]{2}{*}{ Property } & \multicolumn{2}{|c|}{$\overline{\mathrm{Rf}}$} & \multirow[t]{2}{*}{$\mathrm{Db}$} & \multirow[t]{2}{*}{$\mathrm{Sg}$} & \multirow[t]{2}{*}{$\mathrm{Bh}$} & \multirow[t]{2}{*}{ Hs } \\
\hline & MCDF & CCSD & & & & \\
\hline Ground state & $6 d 7 s^{2} 7 p$ & $6 d^{2} 7 s^{2}$ & $6 d^{3} 7 s^{2}$ & $6 d^{4} 7 s^{2}$ & $\begin{array}{l}6 d^{5} 7 s^{2} \\
(90 \%)\end{array}$ & $\begin{array}{l}6 d^{6} 7 s^{2} \\
(91 \%)\end{array}$ \\
\hline Excited state & $6 d^{2} 7 s^{2}$ & $6 d 7 s^{2} 7 p$ & $6 d^{2} 7 s^{2} 7 p$ & $6 d^{3} 7 s^{2} 7 p$ & $6 d^{4} 7 s^{2} 7 p$ & $6 d^{5} 7 s^{2} 7 p$ \\
\hline Excitation energy / eV & 0.5 & 0.3 & 1.9 & 1.64 & 0.79 & 0.63 \\
\hline Ionization energy / eV & 6.5 & 6.01 & 6.89 & 7.10 & 7.7 & 7.6 \\
\hline
\end{tabular}

For the references see table 2 in Reference 3.

orbitals (AO) in case of an atom or molecular orbitals (MO) in case of a molecule, which themselves are usually constructed in a linear combination of atomic orbitals (LCAO)

$$
\phi_{k}=\sum_{i=1}^{n} c_{i k} \chi_{i}
$$

The resulting matrix equations with the coefficient matrix $\underline{\underline{c}}$, the overlap matrix $\underline{\underline{S}}$ and the Fock matrix $\underline{\underline{F}}$ are often called Roothaan LCAO-MŌ-SCF method

$$
\underline{\underline{F}} \underline{\underline{c}}=E \underline{\underline{S}} \underline{\underline{c}} .
$$

These equations are the basis for most practical computational methods in Quantum Chemistry.

2.4. Choice of a Basis Set. In all of these calculations usually the single particle wavefunctions $\phi_{i}$ are expanded in either Gaussian-type orbitals (GTO)

$$
\chi_{u v w}=N x^{u} y^{v} z^{w} \exp \left(-\alpha r^{2}\right)
$$

where $u, v, w$ are the non-negative integers and $\alpha$ is the positive orbital exponent, or

Slater-type orbitals (STO)

$$
\chi_{n l m}(r, \theta, \varphi)=N \cdot r^{n^{*}-1} \exp \left(-\frac{Z^{*}}{n^{*}} \frac{r}{a_{0}}\right) Y_{l m}(\theta, \varphi)
$$

which do not represent the inner orbitals well because they do not have the right number of nodes, or numerical atomic orbitals which are generated from numerical atomic structure calculations. These orbitals have the correct number of nodes and show a correct asymptotic behavior.

2.5. Correlation Energy. The problem is that HartreeFock takes into account the interelectronic repulsion only in an average way. To account for this deficiency one has to include the so-called "correlation energy". There are various methods to account for this:

One possible method is the configuration interaction (CI). Here (in principle) every Slater determinant $\psi_{k}$ which can be constructed from the occupied and unoccupied Hartree-Fock orbitals contributes to the total wavefunction

$$
\Psi=\sum_{k=1}^{\infty} c_{k} \psi_{k}
$$

Depending on the fact if only single (S), double (D), triple (T), etc. excited configurations are included, the method is called CIS, CISD, CISDT, etc.

In the multi-configuration self-consistent field (MCSCF) method a limited number of configuration state functions are used, but not only the expansion coefficients $c_{k}$ are determined. In addition, the single particle wavefunctions $\phi_{u}$ which construct the Slater determinants $\psi_{k}$ are optimized .

Another possibility to treat correlation is the many-body perturbation theory (MBPT). In the Møller-Plesset theory (MP) the Hamiltonian is split into the unperturbed Hartree-Fock operator $H^{(0)}$ and the perturbed rest $H^{(1)}$

$$
\hat{H}=\hat{H}^{(0)}+\hat{H}^{(1)} \text {. }
$$

The most sophisticated description is the coupled-cluster (CC) theory. Other typical quantum chemical methods are e.g. the zero differential overlap approximation, NDDO, CNDO, or any kind of Hückel approximation including the extended Hückel theory. All these methods shall not be discussed here.

2.6. Density Functional Approach. Slater had the idea to use the exchange term in the Hartree-Fock equations as an effective potential. The exchange of a free electron gas is given as $(\alpha=1)$.

$$
V_{X_{\alpha}}=-3 \alpha(3 \rho / 8 \pi)^{1 / 3},
$$

where $\alpha$ is an adjustable parameter, and $X$ refers to the exchange. Thus the one-electron energies and wavefunctions are determined by solving

$$
\left(-\nabla^{2}+V_{c}+V_{X_{\alpha}}\right) \phi_{i}=\varepsilon_{i} \phi_{i}
$$

In principle, this method is also exact. It is based on the Hohenberg-Kohn theorem which shows that the ground state density $n$ corresponds uniquely to a given external potential. In general the total energy of a system is

$$
\mathcal{E}[n]=T[n]+\mathcal{E}^{e x t}[n]+\mathcal{E}^{h}[n]+\mathcal{E}^{x c}[n]+E_{\alpha \beta},
$$

where $T[n]$ is the kinetic energy, $\mathcal{E}^{e x t}[n]$ the electron-nucleus interaction, $\mathcal{E}^{h}[n]$ the direct electron-electron interaction, $\mathcal{E}^{x c}[n]$ the exchange-correlation part of the electron-electron interaction and $E_{\alpha \beta}$ the nucleus-nucleus interaction energy. The problem with this method is that one does not know the exact $\mathcal{E}^{x c}[n]$, but only approximations.

\section{Relativistic Atomic Calculations}

First relativistic atomic structure calculations for atoms became available at the end of the 60s. They led to a first qualitative discussion of the structure of the valence electrons in very heavy elements. However, the quality of these results was only of limited overall predictive power. Later, the development of high-speed computers as well as relativistic atomic codes made it possible to approach the goal of an improved understanding of these very heavy elements with $Z \geq 100$. A summary is given in the review Reference 3.

An example for these calculations is presented in Table 1 which summarizes atomic structure calculations for the elements 104-108.

\section{Relativistic Molecular Calculations}

A new period in the study of elements started when it became possible to perform calculations of small molecules which contain one or more atoms with a charge number above $Z=100$. To our knowledge, the first application with the newly developed discrete variational method was a calculation of $(110) F_{6}$ in 1978. ${ }^{13}$ In the 80 s this method has been extended considerably. During the last years, calculations of improved wave functions became possible even for more complex molecules. Since then a relatively large number of already complex molecules of all elements have been calculated. In order to compare the quality of the various methods and approximations discussed here one should compare those for the same molecule. Unfortunately, 
TABLE 2: Results from various calculations of diatomic $\mathbf{A u}_{2}$.

\begin{tabular}{clccc}
\hline Ref. & Method & $R_{e} /$ a.u. & $D_{e} / \mathrm{eV}$ & $\omega_{e} / \mathrm{cm}^{-1}$ \\
\hline 4 & P-HFS & 4.62 & 2.52 & 201 \\
5 & SRPP-HF & 4.93 & 0.83 & 160 \\
5 & SRPP-MP2 & 4.64 & 2.53 & 205 \\
5 & SRPP-CCSD & 4.74 & 2.00 & 185 \\
5 & SRPP-CCSD (T) & 4.73 & 2.22 & 182 \\
6 & cp-CCSD (T) & 4.70 & 2.19 & 187 \\
7 & CASSCF-MRSDCI & & 2.00 & \\
8 & ZORA-GGA & 4.76 & 2.26 & 174 \\
9 & ZORA-GGA & 4.75 & 2.31 & 178 \\
10 & ZORA-GGAI & 4.76 & 2.53 & 177 \\
10 & ZORA-GGA2 & 4.76 & 2.26 & 174 \\
11 & ZORA-GGA & 4.75 & 2.33 & 183 \\
our & 4-comp. RGGA & 4.88 & 2.50 & 169 \\
12 & exp & 4.67 & 2.29 & 191 \\
\hline
\end{tabular}

there is no such system besides the diatomic $\mathrm{Au}_{2}$ where we summarize the binding energy $D_{e}$, bond distance $R_{e}$, and vibrational frequency $\omega_{e}$ from several calculations. A few references are given in Table 2.

\section{Conclusion}

Many features of the transactinide elements are now relatively well understood. The statement that relativistic effects play a dominant role in the prediction of physical quantities and chemical behavior is now well established. Extended calculations will certainly change details of the predictions for specific elements in the future, but not the gross structure. The quality of molec- ular calculations has still to be improved, but for a number of elements one already knows large details of their chemical behavior.

\section{References}

(1) B. Fricke, W. Greiner, and J. T. Waber, Theor. Chimica Acta (Berlin) 21, 235 (1971).

(2) P. Pyykkö and J. P. Desclaux, Chem. Phys. Lett. 50, 503 (1977).

(3) V. Pershina and B. Fricke, Heavy Elements and Related New Phenomena, Vol. I, edited by W. Greiner and R. K. Gupta (World Scientific, Singapore, 1999), p. 194.

(4) T. Ziegler, J. G. Snijders, and E. J. Baerends, Chem. Phys. Lett. 75, 1 (1980).

(5) R. Wesendrup, J. K. Laerdahl, and P. Schwerdtfeger, Chem. Phys. Lett. 110, 9457 (1999).

(6) B. A. Hess and U. Kalder, J. Chem. Phys. 112, 1809 (2000).

(7) K. Balasubramanian, P. Y. Feng, and M. Z. Liao, J. Chem. Phys. 91, 3561 (1989).

(8) E. v. Lenthe, E. J. Baerends, and J. G. Snijders, J. Chem. Phys. 101, 9783 (1994).

(9) E. v. Lenthe, J. G. Snijders, and E. J. Baerends, J. Chem. Phys. 105, 6505 (1996).

(10) M. Mayer, O. D. Häberlen, and N. Rösch, Phys. Rev. A 54, 4775 (1996).

(11) W. Liu and C. Wüllen, J. Chem. Phys. 113, 2506 (2000).

(12) L. L. Ames and R. F. Barrow, Faraday Soc. 63, 39 (1967).

(13) A. Rosen, B. Fricke, and T. Morovic, Phys. Rev. Lett. 40, 856 (1978). 